\title{
Involúcrame y entenderé. El gran reto de no estandarizar, sino, subir el estándard
}

\section{Buey Fernández, Mercedes [ver currículum del autor, docente de la Facultad de Diseño y Comunicación]}

\section{Resumen:}

Los sectores más influyentes impulsan cambios de fondo en materia de negocios, lo que repercute de forma positiva en el mundo del diseño.

Estos, obligan a re imaginar la fabricación y repensar la posición del diseñador, no sólo frente al producto terminado, sino, a repensar su rol predominante en la cadena de intercambios culturales y de bienes. Ante la emergencia de un mundo dominado por lo visual, se impone una nueva manera de pensar y abordar el diseño. Sin embargo, por paradójico que parezca, la mirada está puesta en la invisibilidad del producto y en características que no se observan hasta el momento de uso; en la experiencia de empleo de un determinado producto, y no en las características físicas con las cuales todos los diseñadores dotan a aquellos. Importan las posibilidades de un producto inmerso en determinado contexto, y no sus características en sí.

Palabras clave:

Diseño - negocios - globalización - glocalización - conectividad - actualización - diseño de experiencias - diseño de producto.

Introducción

El auge actual de los entrepreneurs ha generado una abundante literatura, así como la publicación de casos y la proliferación de cursos y seminarios que son, en resumidas cuentas, no más que la retroalimentación misma de ese auge. Consecuentemente, la carencia de una teoría general, que abarque de manera calificada, con definiciones universales y posturas atractivamente contrapuestas, del tema que se trata, es quizá una de las 
principales oportunidades que se aprovecha hoy en día. Esto potenció los estudios dedicados al desarrollo humano, basados en el diseño y rediseño eficaz de productos y procesos productivos, dando cuenta de la impronta estratégica necesaria garantizar tal desarrollo.

Probablemente, el beneficio más importante que se pueda rescatar de dicha bibliografía, al margen de la teoría dura, sea el de la experiencia que cada profesional pudo adquirir, demostrando ahora que puede desenvolverse y posicionarse de manera segura para enfrentar cualquier contexto laboral, aprendiendo los negocios de la vida real. Esta tendencia se verifica cada vez más real y se vuelve cada vez más notoria, sobre todo en el contexto actual, en el que las profundas transformaciones sociales, culturales, tecnológicas, económicas y ambientales demandan una reflexión consciente en torno a esa actitud emprendedora y personalidad audaz, como condición sine qua non y motor para el desarrollo humano. Los sectores más influyentes, impulsan hoy cambios de fondo en materia de negocios y esto repercute de forma positiva en el mundo del diseño. Estos cambios obligan a re imaginar la fabricación y repensar la posición del diseñador, no sólo frente al producto terminado, sino, a repensar su rol predominante en la cadena de intercambios culturales y de bienes. A esta altura, no son tan nuevos los enfoques al desarrollo que hacen énfasis en el "carácter instrumental del acceso a bienes y servicios, concibiéndolos únicamente como un medio para poder alcanzar un plan de vida o una realización individual plena". (Bilbao Ubillos, 2009) Sin embargo, en este contexto, es nuevamente válido rescatar dichas aproximaciones como aportes necesarios para una reflexión total acerca del quehacer profesional del diseñador. Tales perspectivas, como se ha señalado en otras oportunidades, son:

Determinantes para avanzar hacia una nueva indagación del concepto, en la que el desarrollo económico constituye una condición necesaria pero no suficiente para explicar el grado de avance de una sociedad y en la que el trabajo constante de diseño y producción son el desarrollo en si mismos, y los objetos producidos son en realidad, el resultado más directo de esa experimentación activa y actitud de cambio. (Buey Fernández, 2014)

En tiempos de crisis el mercado se intensifica y el espíritu emprendedor se reaviva. El caos da lugar al surgimiento de nuevos procesos y productos. Los océanos se vuelven azules para los diseñadores obligándolos a salir de su zona de confort, a tomar la iniciativa, a desarrollar nuevas capacidades y a dar el máximo de cada uno para establecer procesos que logren ampliar los límites de los mercados actuales. Es momento de explorar zonas inexploradas, centrarse en ideas globales, mejorar las circunstancias dadas y generar productos que se vuelvan verdaderos negocios. Esta actitud, es la actitud fundamental para el cambio social. (Kiyosaki, 2011) En este marco, resulta interesante el análisis del potencial de expansión global que tienen los productos, y la evolución que existe en materia de conectividad, que exige a su vez a la actualización peramente e impulsa un consecuente desarrollo cultural y generacional. Se naturalizan términos como glocalización y networking, y se encuentran más productos resultantes de voluntades colectivas, que producciones individuales de diseñadores estrella. Se cree aún en el diseño como factor de cambio, y se camina hacia una nueva sociedad a través de prácticas creativas que permitan que la vida de las personas sea sencilla, divertida, cómoda y productiva. La disciplina siempre ha ejercido un papel revolucionario en este sentido, y verdaderamente transformador, a pesar de los diferenciados niveles de resultados. Aún así, es necesario remarcar que, en las sociedades amenazadas por sus propios hábitos, la creatividad en el diseño no alcanza. En la actualidad, el contexto latinoamericano por ejemplo, se muestra volátil en materia económica e inestable en lo que a política se refiere. De este modo, la sociedad se ve obligada a pensar de manera estratégica y de mediano plazo. (El economista, 2015) En términos de desarrollo, entendido como se dijo anteriormente, se puede decir que entonces ya no alcanza con aceptar resultados conocidos, sino que es necesario plantear cada vez más ensayos donde uno pase, como dice Aicher 
(2001, p. 137), "del pensar al hacer y, en ese hacer, aprender de nuevo a pensar". La mejor teoría es una buena práctica, se sabe decir y en ese sentido, hacer que las cosas sucedan, es la premisa actual. Dime y olvidaré, muéstrame y podría recordar, involúcrame y entenderé, reza un proverbio chino que sirve para ilustrar el estado del arte del diseño, su enseñanza y su práctica profesional. En tiempos de desequilibrio y reajuste, en los que nuevas necesidades afloran y se convierten en las mejores oportunidades de mercado, liberarse de cualquier estructura, tomar las riendas del negocio y hacer foco en lo fundamental, son las claves para posicionarse de la mejor manera. Sin que parezca en principio un tratado de economía, lo importante del presente escrito es posar la mirada en la variable de negocio, de rentabilidad y de oportunidad de los proyectos, que nada tiene que ver con el dinero en sí. Como se ha dicho, no se trata de los productos puntualmente, si no, de la lógica de esos productos. Los mejores profesionales surgen en tiempos de crisis. "Cuando los vientos de cambio soplan, algunos construyen refugios y se ponen a salvo, otros construyen molinos y se hacen ricos". En el marco del escenario actual, esa frase adjudicada a Clos Möller, experto asesor empresarial y fundador de una de las mayores consultoras del mundo, tiene cada vez más pertinencia. Los verdaderos diseñadores serán aquellos audaces y creativos que logren construir su molino de la manera más ingeniosa. Refugios o molinos; innovación o muerte, pareciera ser entonces la hipótesis signante de todo proyecto actual. En términos creativos la innovación no es únicamente lo que hacen los diseñadores, sino, lo que los usuarios adoptan, utilizan y, al final, validan realmente. Es la experiencia del usuario final lo que importa. Desde la práctica por ejemplo, se puede afirmar que las organizaciones de diverso tamaño están cada vez más convencidas que la comunicación, la construcción de marca, la conceptualización de los proyectos y el redescubrimiento de la simpleza, son parte importante de su estrategia general. (Costa, 2015) Ante la emergencia de un mundo dominado por lo visual, se impone una nueva manera de pensar y abordar el diseño. Sin embargo, por paradójico que parezca, la mirada está puesta en la invisibilidad del producto y en características que no se observan hasta el momento de uso; en la experiencia de uso de un determinado producto, y no en las características físicas con las cuales todos los diseñadores dotan a los productos. Importan las posibilidades de un producto inmerso en determinado contexto, y no las características del producto en sí.

En tiempos en los que la innovación y el diseño han dejado de ser opciones, para constituir variables indispensables, los diseñadores actuales deben dar muestra de su capacidad creativa e iniciativa colaborativa, como características ineludibles para contribuir de manera activa al desarrollo profesional dentro del campo disciplinar, conduciendo a las sociedades a un cambio de mentalidad e incitando a través de sus productos y servicios, a nuevas formas de desarrollarse y vincularse. En consecuencia, es un acierto analizar la estrecha relación entre la variedad temática, metodológica y conceptual, existente en los proyectos actuales de diseño, con esta búsqueda de la flexibilidad que demanda el campo disciplinar a los profesionales. La mirada está puesta hoy en diseñadores que se nuclean por proyectos y trabajan en colaboración, a partir de clientes que solicitan el trabajo, pero que no cierran las posibilidades a un solo proveedor sino que, consideran que los sujetos y las prácticas son más importantes que los métodos en si mismos y apuestan a la pluralidad de miradas y el trabajo colaborativo -networking- para garantizar la evolución. Esa evolución no es únicamente cultural y generacional; la transformación tecnológica se muestra más profunda que la social y en función de eso, se observa hoy un claro cambio de paradigma. La modalidad home office, las reuniones vía skype, la difusión de mensajes hangout y las conversaciones fotográficas a través de snapchat, permiten compartir archivos en tiempo real, mantener negocios online a costes despreciables e incluso gratis, expandiendo los límites del término conectividad. No se descubre nada al afirmar que el mundo se ha complejizado, que la vida de hoy es más estresante y los contextos laborales ponen la vara cada vez más alta. No se habla ya de una era industrial 
si no de, una era de la información con trabajadores del conocimiento. Los retos que se presentan son de magnitudes desconocidas y de un tipo completamente distinto al que uno pudiera imaginarse alguna década atrás. Estos cambios drásticos en la sociedad y las consecuentes réplicas del mercado global y digitalizado obligan a la actualización constante y a trabajar en estructuras que permitan avanzar tan rápido como lo hace la tecnología. Aún así, por momentos, la barrera cultural es muy grande y el común de la gente desconoce las herramientas. "Cuando cambia la infraestructura se tambalea todo" afirman (Covey, 2003). No obstante, los retos son universales y las soluciones a los problemas se basan, y se basarán siempre, en principios universales, comunes a todas las sociedades. No se inventan hoy aquí, simplemente es primordial identificarlos y organizarlos en un marco secuencial. En última instancia el éxito de cualquier esfuerzo depende de siempre hacer las cosas en armonía con los principios asociados al éxito que se persigue (Covey, 2003). La clave de diseño está pues, en las investigaciones críticas y cualitativas por sobre las cuantitativas del estado de la cuestión. Tomando como base el trabajo de Tom Peters, se reconoce que la infraestructura tecnológica es hoy fundamental para el desarrollo de cualquier negocio. Ésta acelerará las tendencias, tanto las buenas como las malas; pero, por eso mismo, el factor humano será cada vez más importante también. Los puestos no abundan, pero en el nuevo orden mundial, donde el software se está haciendo cargo del trabajo mecánico y repetitivo, la alta tecnología sin el toque humano no funciona, y cuanto más influyente sea esa tecnología más importante es el factor humano que controla es tecnología. Hoy nadie sobrevive sentado detrás de un escritorio realizando una y otra vez la misma tarea esperando resultados distintos cada vez. (2004) En la actualidad, las personas con talento están menos dispuestas a esperar su turno. Para muchos, los nuevos jóvenes profesionales están a disposicipara cambiar el mundo y poseen en muchos casos, más cultura y más conocimientos que sus padres para hacerlo. A lo largo de los próximos años todo lo que se hace y el modo en que se hace va a ser reinventado como resultado de la revolución de las tecnologías, y quienes sepan aprovecharse mejor de este cambio, desafiar el status quo y cosechar los mejores frutos, entregando valores distintivos, se convertirán en los "maestros del talento". (Peters, 2004) Con el objetivo de quebrantar ese sistema rígido bajo el cual se viene moviendo el mundo de los negocios, se ponen en marcha los proyectos más creativos y por consiguiente, se habla hoy de una nueva era de los negocios, lo que suscita cambios estructurales en el modo de ver, entender y aprender el diseño. Se forman hoy los diseñadores del mañana y se habla del capital intelectual, y de la diversidad como clave de ese capital.

De la globalización a la glocalización: nuevas oportunidades de colaboración masiva

El mundo necesita hoy, más que nunca, de creadores, de diseñadores, de profesionales capaces de pensar y repensar las soluciones más creativas a los problemas cada vez más cotidianos. El surgimiento de nuevos líderes y la reinvención de los existentes, resulta imperiosa. La imagen del guía gentil, de personalidad atractiva, agradable y con respuesta para todo, ubicado en la cima de cualquier organigrama, ha quedado desactualizada. El dinamismo y la vertiginosidad del mundo actual, exige respuestas cada vez más rápidas y adecuadas, producidas por "mentes totales". Estas mentes son equilibradas en sí mismas, pero están a su vez conectadas con una multitud de otras mentes totales. Esa conjunción es lo que algunos llaman: "el cerebro global". Ese cerebro global, es el "que pasa a través de las redes, el que teje y desteje en la medida de las necesidades: el que está atento a las necesidades del mundo, en un perpetuo feedback que nada tiene que ver con el pensamiento único". (Martínez-Salanova Sánchez, s/f) Hablar y escuchar hablar de globalidad y globalización con frecuencia, es sencillo. Sin embargo, pocos logran una aproximación que se ajuste al contexto de los modos de vida actuales. La globalidad, la globalización, es compleja y a su vez tan aún en desarrollo que casi pudiera 
decirse que todavía no está ni cerca de encontrar sus límites. El problema para muchos es que manejan paradigmas de la era industrial en plena era de la información y el conocimiento. Lo mismo que jugar un juego nuevo con reglas viejas. En pocas palabras, ese es el gran reto que las mentes globales plantean a la educación actual: el de la "creatividad organizada". (Martínez-Salanova Sánchez, 2002) Durante la era industrial, las instituciones capacitaban a sus estudiantes para ejercer una única profesión por el resto de sus vidas. Ahora, la educación debe ser permanente, y sobre todo, la autoeducación. Esta debe extenderse de por vida si uno quiere mantenerse actualizado. Antes cualquier profesional encontraba su apogeo profesional alrededor de los 40 años. Hoy, probablemente a esa edad se encuentre en decadencia si no se mantuvo activo y actualizado. En la era industrial un título universitario, una capacitación profesional o una tecnicatura eran garantía de un futuro próspero y certificado de estatus económico aceptable. Lamentablemente, hoy, en la era de la información y el trabajador del conocimiento, un título universitario únicamente no es sinónimo de buen empleo o salario. Una buena educación académica hoy no es suficiente. Este cambio no es ni bueno ni malo, es simplemente un cambio, pero si exige flexibilidad, y quien no sea flexible, sufrirá. Los cambios se suceden de manera exponencial y nadie puede permanecer ajeno. La única solución hoy posible es que todos cambien, de la mejor manera, en conjunto con el mundo. Lo único capaz de permanecer es aquello que cambia. Quien no cambia, quien no se adapta, muere. Hace algunos años atrás los cambios se gestaban y sucedían de forma paulatina, hoy, se reciben cambios de manera casi diaria. Suena lógico entonces pensar que lo que asombra hoy no son los cambios, sino, la velocidad en la que estos se suscitan. (Samsó, 2009) Los diseñadores tienen que enfrentarse a los competidores de nacionalidades diferentes con características técnicas, económicas, organizativas y sociales muy variadas. La productividad se proyecta sobre los conceptos de variedad y adaptación al cliente -producto a su medida-. La flexibilidad implica una ventaja competitiva, ya que actúa sobre el producto y sobre el proceso reduciendo el tiempo de respuesta y convirtiendo las necesidades en demandas. En un entorno globalizado, dinámico, la resistencia al cambio durante un período prolongado va a generar la marginación de la empresa y, al final, su desaparición. La empresa se revela como un sistema dinámico no lineal en el que los actores se comunican, interactúan e intercambian información constantemente. La realidad del imperativo global hace necesario considerar sus oportunidades de forma diferente. Ya no se interpretan en términos del mercado local o país, se debe considerar al mundo entero como una oportunidad empresarial. El mundo existe más allá de las grandes empresas y es preciso entender esto para lograr la tan apreciada cosmovisión. La confusión se da al momento de concebir la globalización de un modo liviano y "un tanto casual", reduciendo el término a su acepción cuantitativa, para referirse a fenómenos de gran escala únicamente, dando a entender que interesa únicamente a aquellos actores involucrados en dichos fenómenos, en contraposición a aquellos interesados en proyectos de micro escala o locales. Para muchos, semejante modo de abordar el tema, da lugar a dudosas consideraciones de la cuestión, tales como que de la escala -medida en términos cuantitativos- del fenómeno depende su categorización en global o no, y que en dicha categorización no hay lugar para el análisis cualitativo de los aspectos locales del fenómeno, por ejemplo. Esta tendencia se construye evidentemente, sobre "Ios intentos hartos numerosos por 'internacionalizar' remitiéndolo a desarrollos que implican el triunfo de las fuerzas culturalmente homogeneizadoras sobre todas las demás". (Robertson, s/f) En este sentido, si la mentalidad global moderna es diferente a la internacional, entonces debe ser capaz de solucionar distintos problemas. Estos problemas son los de la glocalización. El término glocalización, fue acuñado en 1980 por Roland Robertson, y nace de la conjunción entre globalización y localización. Según el sociólogo, es el intento de entender las maneras en las que lo global y lo local interactúan para producir una nueva cultura, en la cual las personas y organizaciones estén dispuestas a pensar globalmente y actuar localmente. En este nuevo marco, la respuesta ha de ser indefectiblemente colectiva 
Interdependencia: el poder del trabajo en colaboración

"Ábrete y deja que calen en ti las mejores cosas que hayan hecho los humanos, y luego intenta incorporar esas cosas a lo que estas haciendo" (Jobs, s/f)

"La creatividad, tanto individual como social, es la pieza clave para resolver los problemas que se le plantean a la especie humana". Pero como se marcaba líneas más arriba, con eso solo no alcanza. Las nuevas aproximaciones al término distinguen a la evolución como "el resultado de la solidaridad y la interrelación". En favor de esto, las nuevas tecnologías, las nuevas tecnologías de información y sobre todo la velocidad de las máquinas y la facilidad para buscar y almacenar información, aceleran cualquier proceso intuitivo, incrementando sustancialmente dicha posibilidad de interrelación, ya que en parte la creatividad se da por la cantidad y no la calidad de ideas que es capaz de esbozar el cerebro humano, quien luego las filtra, las vuelve a codificar las utiliza y las relaciona con otras para crear la idea de calidad que se ajuste a las necesidades previstas. Esto se valida gracias a la conexión de mentes que establecen redes a partir de las cuales promueven la aceptación participativa y crítica de la sociedad entera, ya que la creatividad, necesaria para buscar soluciones se hace más eficaz cuanto más solidaria, conectada y global sea. (Martínez-Salanova Sánchez, 2002) La redefinición del diseñador como motor para el cambio social se remonta a tiempo atrás y excede los límites del planeamiento proyectual y su realización. Tradicionalmente la enseñanza del diseño -arquitectura, comunicación, producto o servicio- se planteaba a partir de currículas con módulos independientes, cuyos contenidos se vinculaban ocasionalmente. Las capacitaciones actuales configuran nuevos esquemas profesionales con la interdisciplinariedad como característica principal para el entendimiento de la interdependencia, facilitando la interacción del diseñador con todos los actores del proyecto y permitiéndole percibir el contexto en el que se desarrolla como un sistema. Con un enfoque sistémico, casi asemejados a la teoría autopoiética de Maturana y Varela, los grupos colaborativos se definen como capaces de desarrollar la red de relaciones, procesos u operaciones de forma tal que generan con sus interacciones la misma red que las produce. Estos grupos son capaces de apreciar la inestabilidad como componente vital, definiéndose a ellos mismos y acoplándose a un entorno. Los integrantes del sistema son autónomos -definidos por su autorreferencia- y son a su vez un sistema cerrado en constante autorregulación (Varela y Maturana, 1984). Es gracias al sincero entendimiento de esta relación de dependencia recíproca entre los actores, es que se pueden pergeñar las soluciones más duraderas. Si bien en muchas oportunidades, aunque de manera errada, se da por supuesto que algunas profesiones o expresiones artísticas son más creativas que otras, lo real es que creatividad y el cambio son características inherentes de la existencia humana, y lo que valida o no el grado creativo de una obra no es tanto el producto final sino, el proceso proyectual. Esto es, porque el gran mito de la innovación es que las ideas brillantes surgen directamente, y totalmente formadas, de la cabeza de unos pocos genios, casi como un fruto que cae ya maduro de un árbol. Sin embargo, la realidad es que las más geniales innovaciones provienen de un proceso de examen riguroso a través del cual se identifican las grandes ideas y desarrollaron antes de ser realizada como nuevas ofertas y capacidades. (Brown, 2009) La creatividad, según De Bono, no es un talento. Para el autor, la creatividad es una capacidad humana, un potencial factible de ser desarrollado -aunque no de la misma manera- en todos los seres humanos y por eso, cualquiera puede alcanzar altos niveles de producción creativa. En estos términos, el proceso creativo responde más a un proceso de formulación de hipótesis, verificación de las mismas y comunicación de resultados, que a un don innato del individuo. Es por esto que las mentes más creativas centran sus energías en la experimentación, en el método de ensayos y refutación de hipótesis. No buscan resultados perfectos ni se aferran a la teoría. Se apoyan en 
gráficos conceptuales y técnicas de lluvia de ideas para sostener la teoría de cambio como una constante y como pilar para la permanencia. (De Bono, 1970) Varios autores coinciden en que el desarrollo creativo no constituye únicamente un logro personal sino que, muy por el contrario, es un factor determinante en el desarrollo social en general. "Si el hombre no fuera creativo viviríamos aún en las cavernas" opina De la Torre, y nada más verdadero que eso. Sin mentes creativas, aunadas por fines comunes, no hubieran sido posibles los desarrollos científicos y culturales de la humanidad. (2003, p. 28) Esa experiencia se hace cada vez más rica cuando se hace de manera total, integrando experiencias de otras personas, pensando en circuitos y trabajando en redes. La creación de equipos interdisciplinares y el trasvase de información entre sus miembros es vital para garantizar el desarrollo colectivo que es luego lo que favorece el desarrollo individual. Se dice comúnmente que detrás de un hombre hábil, siempre hay otros más hábiles; y he aquí la clave de la era actual. Los productos -los objetos tangibles o aquellos entendidos como el resultado de algún servicio- más influyentes de la vida son el producto sinérgico de muchas mentes. Esta es la explicación de porque los grandes directores de proyecto llenan sus equipos de personas distintas, con personalidades contrapuestas, con características fuera de lo común y abiertas a la confrontación. Transformaciones de esta índole podrían basarse en un sistema teórico fundamental, el de la inteligencia colectiva del filósofo francés Pierre Levy (2004), que concibe la comunicación en red como un proceso de doble vía, que va mucho más allá del lenguaje y que permite poner en común nuestros conocimientos y experiencias, sumando y acumulándolas en una suerte de "superlenguaje de la humanidad", basada en la vitalidad de esas redes de producción, de transacción y de intercambio de conocimientos, lo que es a su vez, condición constitutiva de la inteligencia colectiva. Se plantea ahora un desafío preciso, buscar más, nuevas y mejores soluciones creativas en pos de un bien común. Levy afirma que el desarrollo creciente de colaboración y de alianzas es un testimonio manifiesto de la importancia de las redes de innovación. No es la red la que se vuelve inteligente, sino sus integrantes, por pertenecer a esa red. (Cuaderno urbano, 2015) La innovación constituye en primera medida, trabajar en equipos interdisciplinarios, trasvasar información, hacer circular las ideas y conservarlas como conocimiento, para fundar así la masa crítica y base de conocimientos mayores. Los nuevos directores de proyectos, los líderes actuales, deben entonces garantizar un nivel de desequilibrio justo en cada grupo de trabajo. Son los encargados de formular las preguntas más incómodas, de anticiparse a los problemas y tomar las decisiones más difíciles. Líderes buenos existen muchos, pero buenos líderes son sólo unos pocos. Por tanto, en este sentido, el buen líder es aquel que es capaz de revertir situaciones adversas para que sean productivas en vez de destructivas. Durante mucho tiempo se pensó que las capacidades individuales bastaban para solucionar problemas comunes pero, el concepto de interdependencia es mucho más complejo maduro y avanzado. La interdependencia supone ser capaz y valerse de uno mismo, pero a su vez implica el trabajo en colaboración para lograr mucho más de lo que uno solo podría haber logrado, aún en su máximo esfuerzo. (Covey, 2003) Esta horizontalidad en la interrelación humana da lugar a la participación colectiva y garantiza la socialización de conocimientos y la multiplicación de informaciones de manera organizada. Lo que antes se consideraba individual y espontáneo, hoy es un hecho global que canaliza voluntades individuales aumentando el impacto de los resultados que de ella se desprenden. En esta nueva era del conocimiento y la interdependencia, "los héroes individuales no existen, los únicos que triunfan son los equipos". (Freire, 2004)

Aprendizaje colaborativo: actitud de compromiso

Los cambios en el entorno de la actividad del diseñador son muchos y suceden de manera vertiginosa. El presente escenario laboral, los nuevos modos de vida actuales y esa velocidad de cambio, obligan a todos los 
actores del sistema a revisar sus habilidades para poder seguir el ritmo. La vida no depende los conflictos que uno enfrenta, sino, del modo en que uno los enfrenta. Qué haga o no haga uno con esos conflictos, es lo que marca la diferencia; y quien logre con determinación comprometerse, y se destaque por ese nivel de compromiso, triunfará por sobre aquellos que se involucren simplemente. Si uno es su compromiso, deja ser sus circunstancias. Y en esa búsqueda, el liderazgo educativo tiene que ser una prioridad; y la construcción colectiva y colaborativa del conocimiento, instrumento principal para el cambio. Se considera al compromiso como "el fenómeno esencial para coordinar acciones con otros" (Patricia Hashuel, 2002). Esta es la piedra basal de cualquier tipo de cambio. Se ha comprobado que es básico en el éxito de cualquier proyecto, sin importar su naturaleza. El compromiso por innovar tiene que ver con la combinación balanceada de pequeñas disrupciones, con crear algo que no existía hasta ese momento y con una revalorización de lo existente (Levy Yeyati, 2016). Guarda estrecha relación con la posibilidad de crear nuevas prácticas en nuestra manera de hacer y establecer nuevas interpretaciones en nuestra manera de pensar de forma de hacer que nuestros compromisos sean consistentes con esa nueva realidad que queremos crear (Patricia Hashuel, 2002). Es la eterna lucha entre la indeterminación y el efecto desestabilizador; la plasticidad versus la cristalización. En definitiva, uno sabe que si quiere lograr algo que nunca tuvo, tiene que hacer algo que nunca hizo Es notoria hoy la necesidad que existe en torno a la adecuación de propuestas educativas para las partes involucradas en el sistema. Y, aunque, las circunstancias externas y el contexto limitan el desempeño, la posibilidad de diseñar el futuro está en mano de los educadores de hoy. El interrogante contemporáneo a nivel educación es entonces: ¿se está enseñando hoy lo que lo que se necesitará para afrontar la realidad laboral del mañana? ¿Dónde y cómo se aprende? Quizás la respuesta a dicho interrogante no sea del todo agradable y si bien aún en la mayoría de las aulas aún se enseña a retener y repetir contenido enciclopédico, es importante desatacar que la investigación educativa actual, da cuenta de la fuerte tendencia hacia una acción innovadora, un pensamiento enfocado y el auto-disciplinamiento como herramientas válidas para el fortalecimiento de docentes, con estratégicas de enseñanza más activas. Si la tendencia profesional actual indica que expandirse de manera glocal, a partir del trabajo colaborativo y gracias a la generación de redes de conocimiento, es la respuesta a los desafíos del nuevo siglo, pues el modo en que enseñamos y aprendemos debe actualizarse también. Es por esto que, frente al surgimiento de nuevas perspectivas en el estudio, el aprendizaje y el ámbito laboral, se considera necesario discutir varios aspectos que han dominado la teoría educacional hasta el momento. A pesar de la heterogeneidad de miradas y más allá de la diversidad de abordajes en torno al tema, existe un cierto consenso: "la educación del futuro tendrá que ser flexible, inclusiva, de calidad y adaptable a un paisaje que no cesará de cambiar" (Rua, 2016). Muchos especialistas del área concuerdan en que como docentes, es necesario trabajar en una educación "integral, transversal, contextualizada y que de cuenta de los desafíos futuros que se encontrarán los estudiantes a medida que crecen" (Rua, 2016). Se propone una descentralización de la mirada sobre los procesos individuales y se toma en consideración la importancia de los contextos y circunstancias dadas en torno al estudiante, como elementos de análisis en el camino del aprendizaje ya que estos tienen incidencia directa en los procesos de aprendizaje. En ese punto radica el primer desafío, atender al conjunto de interrelaciones e interacciones recíprocas que se genera entre los estudiantes, y los efectos que dichas interrelaciones puedan tener sobre los procesos. (Elichiry, 2001) Los seres humanos son seres sociales, y este es un axioma bien conocido. Los sujetos se pasan gran parte del día interactuando con sus semejantes, bien sobre la base del uno a uno o agrupados de manera diversa. El discurrir de la vida está marcado, al menos en gran medida, por el rango de esas habilidades sociales. Esas relaciones interpersonales tienen un rol fundamental en el desarrollo del individuo ya que ayudan a progresar profesional y socialmente a cada uno, determinando muchas facetas de la vida. Si los sujetos no logran una vida social feliz, difícilmente lograran una vida feliz en general. (Caballo, 1993) 
El nuevo siglo es testigo de notables logros con respecto al dominio de habilidades técnicas específicas.

Procedimientos de entrenamiento sofisticados son hoy normales, y encontrar profesionales altamente competentes suele ser tarea sencilla. Aun así, el comportamiento social es inadecuado. Por esta razón, la misión como docentes es asumir con decisión el rol de líderes. Aún cuando el escenario se presente confuso, o el trabajo con creatividad e imaginación sea tarea difícil, aceptar dicha responsabilidad de liderazgo es trascendental para pensar al alumno fuera del aula y después de la vida escolar, si esto no sucede, pocas son las posibilidades de crecimiento que existen. El énfasis de estudio sobre las habilidades sociales, sobre el grado y la calidad de las interacciones, lo que va a definir a esas habilidades como núcleo de las relaciones interpersonales, y va a constituir un campo de estudio con grandes posibilidades de aplicación práctica. (Caballo, 1993)

Las habilidades sociales, entonces, se definen como "los modos de saber hacer en la acción con los demás", y "conforman un nexo de unión entre el individuo y su ambiente". (Caballo, 1993, p. 13) Este tema constituye una problemática poco novedosa, pero siempre vigente frente a la necesidad de desenvolverse en el medio, y en relación a las características inherentes de los contextos profesionales actuales. Pero entonces, ¿por qué cuesta tanto la innovación? Quizás, sea porque cada vez que se piensa en innovar en educación, se piensa en un cambio copernicano, un giro de 180 grados, un cambio radical, un borrón y cuenta nueva que venga a desbarrancar años de aportes a la educación. Sin embargo, no debiera ser más que un pequeño cambio estratégico. Muchas veces se diseñan las propuestas de innovación alejados de los contextos de acción sin considerar los recursos los recursos y trayectorias existentes o las necesidades reales, cuestión que avizora un fracaso y desinterés (Masnatta, 2016). Los docentes se muestran cansados frente a la búsqueda de la nueva solución mágica, y lo más difícil es unir a las partes como en un engranaje y, sobre todo, que todos se comprometan y crean que son parte de un proyecto social más grande. Es un error pensar que lo que uno enseña es mero contenido. Para innovar es necesario modernizar la enseñanza, y para esto hay que actualizar al docente pero, modernizar formatos no es eliminar el aula. Pero, ¿implica esto cambiar radicalmente la forma en que se viene enseñando? La respuesta es: depende. Flexibilizar contenidos no es jubilar la educación formal. Hay momentos en los que la teoría clásica sobre la cual se vienen construyendo los modelos de transmisión de conocimientos van a ser aún útiles por la naturaleza del concepto a transmitir o el momento en el que se transmite; y momentos en los que será necesario redefinir las estrategias de enseñanza y aprendizaje, gestionando de manera habilidosa la inteligencia colectiva. Se innova por ejemplo incorporando medios virtuales, convirtiendo los trabajos prácticos en talleres entrenando la aplicación de conocimientos a la solución de problemas (Levy Yeyati, 2016) y promoviendo la alternancia de procesos abductivos, inductivos y deductivos, que generen situaciones macro grupales, grupales e individuales, generando a su vez espacios de autoaprendizaje. El docente, en todos los casos, continúa siendo una guía y un facilitador de ese proceso de aprendizaje, siendo el agente principal en la transformación de la información en conocimiento. La influencia del medio ambiente adquiere una importancia cada vez mayor y el problema radica pues en descubrir cómo opera. El medio no modela la conducta imponiéndose a un sujeto pasivo. El tema central es que son los sujetos los que activamente buscan la interacción con el medio. A partir de esa interacción, el registro que indefectiblemente se hace de la situación no es pasivo, el sujeto lo interpreta, y es esa interpretación de los hechos lo que afecta y modela su aprendizaje, no el acontecimiento en sí. (Echichiry, 2001, p. 129) La construcción intelectual no se realiza en el vacío, sino en relación con su medio circundante. El desarrollo intelectual, entonces, procede del aprendizaje colaborativo, es un proceso activo de doble vía. Este método tiene vida propia cuando el sujeto trasciende al contexto educativo y hace uso del recurso en su vida diaria. El aprendizaje colaborativo implica 
mucho más que una forma de agrupamiento físico; se encuentra entre las técnicas más efectivas para la mejora de la efectividad de los aspectos de la interdependencia. Desde los primeros inicios como entrenamiento, existen, con un mismo fin, dos tipos de compromiso en esto del aprendizaje colaborativo y el desarrollo de las habilidades sociales. En primer lugar, el compromiso primordial es aquel que se establece con uno mismo, persona interesada en adquirir un conocimiento, analizarlo e internalizarlo de tal manera que lo hace suyo para siempre. En segunda instancia, es el compromiso con el conjunto de personas que conforman el entorno cercano de aprendizaje (Montgomery, 1794). Es decir, los intervinientes en el acto de aprendizaje. Aquí es importante destacar que, ningún compromiso grupal puede darse si no es, a partir de un compromiso individual, consigo mismo. El grupo ofrece una situación real de aprendizaje con un contexto menos intimidante, un alto grado de apoyo y un mayor rango de retroalimentación, en la que ya no sólo es importante la calificación individual sino, las implicancias grupales que tiene cualquier acto individual. Al igual que cuando se habla de equipos profesionales, en el aprendizaje colaborativo y en colaboración, el éxito del grupo depende del aprendizaje individual. La clave para lograr esto es que cada uno perciba su rol de ayuda al aprendizaje de sus compañeros también. De ayuda no solamente a la culminación de una tarea individual sino, su rol como motivadores de compromiso, para consigo mismo y para con el grupo en general. (Revista digital: novedades en línea, 2014) Las zonas de desarrollo próximo (Vygotsky, 1978) de los nuevos profesionales se están avivando de manera muy sensible, y las teorías clásicas de aprendizaje parecen quedar pequeñas frente al auge de las nuevas tecnologías de la información. Las redes sociales, las plataformas de código abierto, los blogs y las enciclopedias de construcción colectiva, obligan a reinterpretar el alcance de las teorías y definiciones en torno al aprendizaje social, colaborativo y de desarrollo de habilidades. Las posibilidades de pensar y re pensar la educación son infinitas, y las chances de construir conocimiento, inmensas. El aprendizaje colaborativo o social, es mucho más que el aprendizaje a través de redes sociales, o que la construcción de conocimiento y socialización de la información a través de ellas. Y reducir el aprendizaje social a un concepto tal, sería caer en reduccionismos simplistas. Sin embargo, es ineludible el hecho de que estas impulsan el cambio. La conectividad en tiempo real brinda la posibilidad de almacenar y revisar conocimientos ya adquiridos para generar a su vez nuevos conocimientos factibles de ser publicados, editados y re concebidos de manera indefinida. El análisis general esbozado en este escrito demuestra que los profesionales se están organizando de manera intuitiva para alcanzar objetivos, aunque el marco para dicha organización no sea propuesto desde los niveles iniciales de la educación. Frente a este nuevo panorama social, uno puede darle la espalda y seguir construyendo muros, o puede sumarse a la corriente aperturista y abrir la mente a estas nuevas formas de interacción y comunicación planteadas. Los nuevos modos de aprendizaje social acercan la teoría a definiciones más modernas de aprendizaje y ubican a los docentes en un nuevo lugar, como facilitadores del aprendizaje pero no como únicos productores de conocimiento. El reto moderno es generar ecosistemas de interacción abierta en entornos protegidos a partir de consignas colectivas y entrenamientos asertivos.

Listado de referencias bibliográficas

Aicher, O. (2001). Analógico y digital. Barcelona: Editorial Gustavo Gili S.A. Brown, T. (2009). Change by design. How design thinking transforms organizations and inspires innovation. New York: Harper Collins. Buey Fernández, M. (2014). Desarrollo activo. Pensar al hacer. Buenos Aires: Universidad de Palermo. Caballo, V. E. (1993). Manual de evaluación y entrenamiento de las habilidades sociales. Madrid: Siglo veintiuno editores. Costa, J. (Junio 2015). Internet. Revista oficial del dircom. Imagen y comunicación. Paradigma dircom. Nuevos lineamientos desde el pensamiento de Joan Costa. Paradigma dircom. Nuevos lineamientos desde el 
pensamiento de Joan Costa. 66, 18-19. Covey, S. R. (2003). Los 7 hábitos de la gente altamente efectiva. Buenos Aires: Paidós. Cuaderno urbano (2015). vol.18 no.18 Los espacios de la innovación urbana (2015). Recuperado el 5/11/15 de http://www.scielo.org.ar/scielo.php?pid=S1853-36552015000100008 \&script=sci_arttext De Bono, E. (1970). El pensamiento lateral. Manual de creatividad. Buenos Aires: Paidós. De la Torre, S. (2003). Dialogando con la creatividad. De la identificación a la creatividad paradójica. Barcelona: Octaedro Ediciones. El economista (2015). La pregunta emprendedora del fin de ciclo. Los desafíos de un entorno complejo son proporcionales a las oportunidades. (20 de mayo de 2015) [Revista en línea] Recuperado el 2/1/16 de: http://www.eleconomista.com.ar/2015-05-la-preguntaemprendedora-del-fin-de-ciclo/ Elichiry, N.E. (2001) (comp.) ¿Dónde y cómo se aprende? Temas de psicología educacional. Buenos Aires: Juve Ediciones. Freire, A. (2004). Pasión por emprender. De la idea a la cruda realidad. Buenos Aires: Aguilar. Hashuel, P. (2002). Compromiso. Recuperado el 5/11/15 de: http://wp.patriciahashuel.net/ archivos/category/2002 Jobs, S. (s/f) en Peters, T. (2004). El talento! Madrid: Ediciones Nowtilus. Kiyosaki, R. T. (2011). The business of 21st century. Editado en Argentina por Santillana Ediciones Generales (2013). Levy Yeyati, E. (21 de febrero de 2016). La nueva educación. Quiénes la están pensando en la Argentina. Buenos Aires: La Nación revista, 34-43. Lévy, P. (2004). Inteligencia colectiva por una antropología del ciberespacio. Washington DC: Bireme - OPS OMS. Martínez-Salanova Sánchez, E. (2002). Propuestas críticas y creativas para vivir en la nueva sociedad mediática. Recuperado el 5/11/15 de: http://www.uhu.es/cine.educacion/crea tividadpropuestas.htm

Martínez-Salanova Sánchez, E. (s/f). Los dilemas y retos en un mundo de comunicación global. Universidad de Huelva. Recuperado el 5/11/15 de http://www.uhu.es/cine.educacion/ articulos/dilemas_comunicacion_global.htm Masnatta, M. (21 de febrero de 2016). La nueva educación. Quiénes la están pensando en la Argentina. Buenos Aires: La Nación revista, 34-43. Maturana, H. R. \& Varela, F. J. (1984). El árbol del conocimiento. Las bases biológicas del entendimiento humano. Santiago de Chile: Grupo editorial Lumen. Montgomery, C. (1974). En Pérez, C. (2013). Aprendizaje colaborativo: una interacción social. Disponible en http://luisorlandolg.blogspot.com.ar/2013/07/aprendizaje-colabo rativo-una_30.html Peters, T. (2004). El talento! Madrid: Ediciones Nowtilus. Revista digital: novedades en línea. (Julio 2014) Internet. Aprendizaje colaborativo (2014) recuperado el 1/11/15 de http://issuu.com/helenpatriciapuertasmogollon/docs/novedades_en___nea/5? e=12678783/8686319 Robertson, R. (s/f). Glocalización: tiempo-espacio y homogeneidad heterogeneidad. Biblitoeca visrtual de ciencias sociales. Disponible en: http://www.cholonautas.edu.pe/modulo/ upload/R\%20Robertson.pdf Rua, M. (21 de febrero de 2016). La nueva educación. Quiénes la están pensando en la Argentina. Buenos Aires: La Nación revista, 34-43. Samsó, R. (2009). El código del dinero. Barcelona: Ediciones Obelisco S. L. Vygotsky, L. S. (1978). Mind in Society [La mente en sociedad]. Cambridge, Mass., Harvard University Press. En Lev Semionovich Vygotsky (1896-1934). París: UNESCO París, UNESCO: Oficina Internacional de Educación), vol. XXIV, nos 3-4, 1994, págs. 773-799. Disponible en: http://www.ibe.unesco.org/sites/default/files/vygotskys.PDF

\section{Abstract:}

And this has a positive impact in the world of design. These sectors require manufacturing to reimagine and rethink the position of the designer; not only the finished product, but to rethink its predominant role in the chain of cultural exchanges and goods. Facing the emergence of a world dominated by visuals, this imposes a new way of thinking and for approaching design. However, paradoxical as it may seem, the focus is put on the invisibility of the product and features that are not seen until the time of use; emphasis is place on the user's 
experience with a particular product, and not on the physical characteristics endowed by designers. They care about the possibilities of a product immersed in a given context and not in its particular features.

Key words:

design - business - globalization - globalization - connectivity - update - design of experiences - product design.

Resumo:

Os setores mais influentes propõem mudanças nos negócios, que repercutem positivamente no mundo do design. Estes coagiram a re-imaginar a fabricação e re-pensar a posição do designer, não somente frente ao produto terminado, senão, a re-pensar seu papel predominante na corrente de intercâmbios culturais e de bens. Ante a emergência de um mundo dominado pelo visual, impõe-se uma nova forma de pensar e abordar o design. Contudo, por paradoxo que pareça, a mirada está posta na invisibilidade do produto e em características que não se observam até o momento do uso; na experiência de uso de um determinado produto, e não nas características físicas com as quais todos os designers dotam a aqueles. Importam as possibilidades de um produto submerso em determinado contexto, e não em suas características.

Palavras chave:

design - negócios - globalização - glocalização - conectividade - atualização - design de experiências - design de produto.

Involúcrame y entenderé. El gran reto de no estandarizar, sino, subir el estándard fue publicado de la página 25 a página39 en Cuadernos del Centro de Estudios de Diseño y Comunicación № 64 